\title{
Light-cured dimethacrylate dental restorative composites under a prism of annihilating positrons
}

\author{
Olha Shpotyuk ${ }^{1, A-D, F}$, Adam Ingram 2,B,C,E, Oleh Shpotyuk 3,C,E,F, Elvira Bezvushko ${ }^{1, A, C, E, F}$ \\ ${ }^{1}$ Department of Pediatric Dentistry, Danylo Halytsky Lviv National Medical University, Ukraine \\ 2 Department of Physics, Opole University of Technology, Poland \\ ${ }^{3}$ Institute of Physics, Jan Długosz University in Częstochowa, Poland \\ A - research concept and design; $\mathrm{B}$ - collection and/or assembly of data; $\mathrm{C}$ - data analysis and interpretation; \\ $D$ - writing the article; $E$ - critical revision of the article; $F$ - final approval of the article
}

\section{Address for correspondence \\ Oleh Shpotyuk \\ E-mail: olehshpotyuk@yahoo.com}

\section{Funding sources}

none declared

\section{Conflict of interest}

none declared

Received on July 25,2017

Reviewed on December 4, 2017

Accepted on December 21, 2017

DOI

$10.17219 / \mathrm{pim} / 81450$

\section{Copyright}

○ 2017 by Wroclaw Medical University

This is an article distributed under the terms of the Creative Commons Attribution Non-Commercial License (http://creativecommons.org/licenses/by-nc-nd/4.0/)

\begin{abstract}
Background. Breakthrough resolutions in current biopolymer engineering rely on reliable diagnostics of atomic-deficient spaces over the finest sub-nanometer length scales. One such diagnostic is positron annihilation lifetime spectroscopy, which probes space-time continuum relationships for the interaction between electrons and their antiparticle (positrons) in structural entities like free-volume defects, vacancies, vacancy-like clusters, interfacial voids and pores, etc.

Objectives. This paper is intended to highlight the possibilities of positron annihilation lifetime spectroscopy as an informative instrumentation tool to parameterize free-volume evolution in light-cured dimethacrylate dental restorative composites exemplified by Charisma ${ }^{\circledast}$ (Heraeus Kulzer GmbH, Hanau, Germany) and Dipo ${ }^{\circledast}$ (Oksomat-AN Ltd, Kyiv, Ukraine).
\end{abstract}

Material and methods. The subjects of the study were the commercially available dimethacrylate-type dental restorative composites Charisma ${ }^{\oplus}$ and Dipol ${ }^{\oplus}$. The analysis used a fast-fast coincidence system of 230 ps resolution based on 2 photomultiplier tubes coupled to $\mathrm{BaF}_{2}$ scintillator detectors and ORTEE ${ }^{\odot}$ (ORTEC, Oak Ridge, USA) electronics to register lifetime spectra in normal-measurement statistics evolving $\sim 1$ million coincidences.

Results. The annihilation process in both composites is identified as mixed positron-Ps (positronium) trapping, where ortho-Ps decaying is caused entirely by free-volume holes in the polymer matrix, and the $2^{\text {nd }}$ component is defined mainly by interfacial free-volume holes between filler nanoparticles and the surrounding polymer. The most appropriate model-independent estimation of photopolymerization volumetric shrinkage in dental restorative composites can be done using averaged positron annihilation lifetime. Partially-constrained $x 4$-term analysis of lifetime spectra is less efficient, giving greater scatter of variance with an additional artiffact of fixed shortest lifetime allowing unresolved mixing in the $2^{\text {nd }}$ component. A meaningful phenomenological description of transformations in Ps and positron-trapping sites under light Curing, which occurs more efficiently in Charisma ${ }^{\circledR}$ than in Dipo $^{\circledR}$ nanocomposites, can be developed at the basis of a semi-empirical model exploring a X3-x2-coupling decomposition algorithm.

Conclusions. A deep understanding of void-evolution processes in dimethacrylate dental composites employing positron annihilation lifetime spectroscopy makes it possible to diagnose, characterize and engineer novel biomaterials for advanced use in medical practice.

Key words: positron annihilation lifetime spectroscopy, dental restorative composites, light curing, dimethacrylate 


\section{Introduction}

Composite biomaterials based on dimethacrylate polymers are currently of strong interest to a great number of scientists in view of their practical medical applications, mainly as promising light-cured dental restoratives. ${ }^{1,2} \mathrm{To}$ day, in due course of the development of $3^{\text {rd }}$-generation biopolymers possessing such features as bioinertness, bioactivity and resorbability, it is of high importance to explore not only their atomic level of structural organization, but also the atomic-deficient or void-relevant level. ${ }^{3} \mathrm{~A}$ necessity to provide highly reliable tools for the technological modification and exhaustive characterization of these structural levels, reaching extremely low nano- and subnanometer length scales, is a pivotal requirement to overall success in the engineering of advanced biocomposites.

One of the breakthrough resolutions of this problem seems to be the diagnostics of atomic-deficient space, which could be quite informative in measuring possibilities, despite the enormous variety in the chemistry of different biomaterials. Positron annihilation lifetime (PAL) spectroscopy is known to be an example of such voidsensitive structural methods. ${ }^{4-9}$ This experimental technique, which probes space-time continuum determination for the interaction between electron $\mathrm{e}^{-}$and its antiparticle (positron $\mathrm{e}^{+}$), is especially sensitive to tiny atomic-deficient entities (like free-volume defects, vacancies, vacancy-like clusters and their complexes, interfacial voids and pores, intergranular boundaries, etc.), which are highly deterministic in the functionality of modern biopolymers, using the recent advantages of nanotechnology. ${ }^{9,10}$ But hitherto, the PAL method has been applied mainly to "purely technical" materials, compounds and polymers, semiconductor and metallic alloys, etc. ${ }^{4,7,8}$ The attempts to use this technique for composite biopolymers have not been very successful so far because of significant complications in the unambiguous interpretation of the obtained experimental data.

In this work, PAL spectroscopy will be comprehensively examined in application to 2 advanced dimethacrylatebased dental restorative composites (DRC), Charisma ${ }^{\circledR}$ (Heraeus Kulzer GmbH, Hanau, Germany) and Dipol ${ }^{\mathbb{R}}$ (Oksomat-AN Ltd, Kyiv, Ukraine), both possessing monomer matrices consisting of BisGMA (bisphenol A-glycidyl methacrylate) and TEGDMA (triethyleneglycol dimethacrylate) modified with a multisized filler having a highly dispersive phase of silica $\mathrm{SiO}_{2}$ glass. ${ }^{11,12}$

\section{Material and methods}

\section{Sample preparation details}

The experimental PAL studies were performed for 2 types of commercially available dental restorative composites (DRC), i.e., Charisma ${ }^{\circledR}$ and $\mathrm{Dipol}^{\circledR}$, which are similar from a structural-chemical point of view (both belonging to resin composites based on dimethacrylate-type polymeric networks), but somewhat different in respect to volumetric shrinkage. The DRC specimens studied were prepared by filling an inner volume of disc-shaped plastic molds of uniform size having $6 \mathrm{~mm}$ in diameter and $2 \mathrm{~mm}$ in thickness. The bottom end surface of the plastic disc was covered by polyethylene slice film, which was separated from the sample along with the outer ring around the disc before the PAL experiments. These batches of non-polymerized DRC samples were respectively marked as Dipol-0 and Charisma-0. Then, part of these DRC samples were polymerized by illuminating their upper surfaces with a standard curing dental wireless LED source (LED.T4, SEASKY, Beijing, China), which emitted light in a $420-480 \mathrm{~nm}$ spectral range with $\sim 900 \mathrm{~mW} / \mathrm{cm}^{2}$ output power density. To normalize the light curing protocol for all DRC specimens, the end of the guide tip from the light source was maintained just above the sample surface at a distance of $7 \mathrm{~mm}$, so that the curing light beam fully covered the sample surface. The overall polymerization duration with this LED source was $60 \mathrm{~s}$ to ensure the deeply polymerized state of each DRC (in accordance with manufacturers' instructions). ${ }^{11,12}$ The photopolymerized DRC batches (5 plate samples in A3 shade separated from the disc-shaped plastic molds) were marked as Dipol-60 and Charisma-60. The DRC specimens were stored dry at room temperature $\left(\sim 20^{\circ} \mathrm{C}\right)$ for 3 days prior to being used in the PAL measurements.

\section{Positron annihilation lifetime spectra measurement}

The experimental PAL spectra were registered with a fast-fast coincidence system of 230 ps resolution based on 2 Photonis XP2020/Q photomultiplier tubes coupled to $\mathrm{BaF}_{2}$ scintillator 25.4A10/2M-Q-BaF-X-N detectors (Scionix, Bunnik, Holland) and ORTEC ${ }^{\circledR}$ electronics (ORTEC, Oak Ridge, USA). To ensure the most reliable measurements, which is a pre-condition of correct decomposition, each PAL spectrum was recorded at $\mathrm{T}=22^{\circ} \mathrm{C}$ and a relative humidity of $35 \%$ in normal-measurement statistics reaching nearly 1 million coincidences. The channel width of 6.15 ps allowed 8000 channels in total. The radioactive ${ }^{22} \mathrm{Na}$ isotope of low $\sim 50 \mathrm{kBq}$ activity prepared from aqueous solution of ${ }^{22} \mathrm{NaCl}$ wrapped by Kapton ${ }^{\circledR}$ foil (DuPont ${ }^{\mathrm{TM}}$, Circleville, USA) of $12 \mu$ m thickness and sealed was used as the source of positrons sandwiched between the 2 tested samples.

Themeasured PALspectrawereprocessed with the LT 9.0 program, ${ }^{13}$ stabilizing an average positron lifetime $\tau_{a v}^{\Sigma}$ as the center of mass of the full PAL spectrum:

$$
\tau_{a v}^{\Sigma}=\sum_{i} I_{i} \tau_{i}
$$

where $\tau_{i}$ and $I_{i}$ denote the positron lifetime and intensity of the corresponding fitting components (the resultant accuracies in lifetime and intensity determination were \pm 0.005 ns and $\pm 0.5 \%$, respectively). 
The best fitting of PAL spectra was achieved via mixed channels of trapping, occurring due to defect-related positron $\mathrm{e}^{+}$traps and bound positron-electron $\mathrm{e}^{+}-\mathrm{e}^{-}$(positronium Ps) states. This task can be solved due to multicomponent fitting of PAL spectra with 3 or 4 negative exponentials under unconstrained (free fitting components) or constrained (most often for some fixed fitting parameters, such as shortest positron lifetime maintained close to $0.125 \mathrm{~ns})^{14-16}$ decomposition procedures, and normalized component intensities $(I=3.4)$ :

$$
\sum_{i} I_{i}=1
$$

\section{Positron annihilation lifetime spectra treatment algorithms}

Because of the repulsive interaction with nuclei of environment, the positron e+ penetrates intrinsic regions of preferentially negative-charged or neutral free-volume structural voids. Therefore, the fitting covers realistic channels caused by positrons $\mathrm{e}^{+}$, which annihilate from delocalized states in defect-free bulk, and those trapped from spatially-extended free-volume defects ( $\mathrm{e}^{+}$trapping) and bound $\mathrm{e}^{+}-\mathrm{e}^{-}$states through "pick-up" annihilation with an electron of surrounding material (Ps decaying).

The canonical 2-state trapping model with only 1 kind of $\mathrm{e}^{+}$-trapping defect can be applied to parameterize the experimental PAL spectrum under the condition of a small contribution from the $3^{\text {rd }}$ and higher components, i.e., ignoring all Ps decaying channels. ${ }^{4,6-8}$ This process is characterized by mean lifetime for $\mathrm{e}^{+}$-trapping $\tau_{a v}{ }^{t r}$, defect-free bulk lifetime $\tau_{b}$, trapping rate in defects $\kappa_{d}$ and fraction $\eta$ of trapped $\mathrm{e}^{+}$, defined in respect to the following equations:

$$
\begin{gathered}
\tau_{a v .}=\frac{\tau_{1} I_{1}+\tau_{2} I_{2}}{I_{1}+I_{2}} \\
\tau_{b}=\frac{I_{1}+I_{2}}{\frac{I_{1}}{\tau_{1}}+\frac{I_{2}}{\tau_{2}}} \\
\kappa_{d}=\frac{I_{2}}{I_{1}}\left(\frac{1}{\tau_{b}}-\frac{1}{\tau_{2}}\right) \\
\frac{\kappa_{d}}{\lambda_{b}+\kappa_{d}}=\frac{\kappa_{d} \tau_{b}}{1+\kappa_{d} \tau_{b}}=\tau_{1} \kappa_{d}
\end{gathered}
$$

In addition to these $\mathrm{e}^{+}$-trapping models, the difference between defect-related and defect-free lifetimes, $\left(\tau_{2}-\tau_{b}\right)$ can be accepted as a signature of size of $\mathrm{e}^{+}$traps in terms of the equivalent number of vacancies, whereas the $\tau_{2} / \tau_{b}$ ratio can be ascribed to the nature of these free-volume defects. ${ }^{4}$ In fact, such a 2 -state trapping model describes $\mathrm{e}^{+}$annihilating from 2 distinct states, these being latticedelocalized and defect-localized states, ignoring back escape of trapped positrons. ${ }^{4,6-8}$

The Ps decaying forms another channel of the PAL spectrum, which is caused by $\mathrm{e}^{+}$annihilating from the Ps state as free particles or interacting with $\mathrm{e}^{-}$from the environment. ${ }^{4-6}$ In the ground state, the Ps exists as para-Ps (p-Ps, antiparallel $\mathrm{e}^{-}-\mathrm{e}^{+}$spins) decaying intrinsically with $2 \gamma$-quanta and character lifetime in a vacuum of $0.125 \mathrm{~ns}$, and ortho-Ps (o-Ps, parallel $\mathrm{e}^{-}-\mathrm{e}^{+}$spins) decaying with $3 \gamma$-quanta and lifetime of 142 ns, these states being occupied with a relative formation rate of $1: 3$. Since $\mathrm{e}^{+}$wave function overlaps with $\mathrm{e}^{-}$outside, the annihilation with such $\mathrm{e}^{-}$having an antiparallel spin decreases their lifetime to $0.5-10$ ns, resulting in $2 \gamma$-rays ("pick-off" annihilation). ${ }^{4,5}$ Two conditions should be satisfied to form Ps, the $1^{\text {st }}$ being a sufficiently high radius of void captured Ps, and the $2^{\text {nd }}$ being low electron density preventing direct $\mathrm{e}^{+}-\mathrm{e}^{-}$annihilation. ${ }^{5}$ The Ps localized in free-volume spaces gives an indication of their mean radii $R$ in terms of long-lived $\tau_{3}$ lifetime (the relative intensity of this component $I_{3}$ correlates with the density of Ps sites) in respect to the Tao-Eldrup equation:

$$
\tau_{3}=0.5 \cdot\left[1-\frac{R}{R+\Delta R}+\frac{1}{2 \pi} \cdot \sin \left(\frac{2 \pi R}{R+\Delta R}\right)\right]^{-1}
$$

where $\Delta R=0.166 \mathrm{~nm}$ is the fitted empirical layer thickness. ${ }^{4,5}$ By fitting the above equation with measured $\tau_{3}$, the $R_{3}$ and corresponding free volumes $V_{f}$ in spherical approximation can be determined. The fractional free-volume $f_{v}$ can be calculated as:

$$
F_{v}=C \cdot I_{3} \cdot V_{f}
$$

using the empirical constant $C=0.0018 \AA^{-3} .^{5}$

Thus, in the case of highly-inhomogeneous substances such as polymers, molecular compounds or composites, the PAL spectra are expected to be composed through a mixed $\mathrm{e}^{+}$-Ps-trapping path.

In case of stronger input from Ps decaying in the x3-term decomposed PAL spectrum (as for many nanocomposites $^{5,9,14-21}$ ), the $\mathrm{e}^{+}$-trapping can be defined in terms of a simple trapping model assuming 2 additive inputs arise from trapped $\mathrm{e}^{+}$and decayed o-Ps states. ${ }^{22}$ This model with 2 additive $\mathrm{e}^{+}$-trapping defects with $\kappa_{d 1}$ and $\kappa_{d 2}$ annihilation rates defined as

$$
\begin{gathered}
\kappa_{d 1}=I_{2}\left(\frac{1}{\tau_{1}}-\frac{1}{\tau_{2}}\right) \\
\kappa_{d 2}=I_{3}\left(\frac{1}{\tau_{1}}-\frac{1}{\tau_{3}}\right) \\
\tau_{b}=\left(\frac{I_{1}}{\tau_{1}}+\frac{I_{2}}{\tau_{2}}+\frac{I_{3}}{\tau_{3}}\right)^{-1}
\end{gathered}
$$




\section{Results and discussion}

The raw PAL spectra were reconstructed from unconstrained $\mathrm{x} 3$-term and partially-constrained $\mathrm{x} 4$-term fitting procedures assuming the shortest lifetime $\tau_{1}$ fixed at the theoretical value of intrinsic p-Ps self-annihilation (0.125 ns). These spectra are depicted at a general background of source contribution for initial and photopolymerized DRC in Fig. 1 and 2, respectively. The narrowrestricted statistical scatters of variance tightly grouped around the 0 -axis testify that the PAL measurements are well described within these fitting procedures, but unconstrained $\mathrm{x} 3$-term decomposition has an obvious preference in view of better goodness of fit.

The best-fit parameters of the detected PAL spectra decomposed in 3 negative exponentials are given in Table 1. The numerical values of $\mathrm{e}^{+}$-trapping and Ps-decaying modes for these DRC calculated with respect to the above formalism of equations (1)-(8) ignoring contribution from o-Ps decaying are presented in Table 2. The similar results obtained with equations (9)-(11) assuming 2 additive $\mathrm{e}^{+}$-trapping defect states are summarized in Table 3, are given for the measured PAL spectra decomposed in 3 negative exponentials. The respective results for these
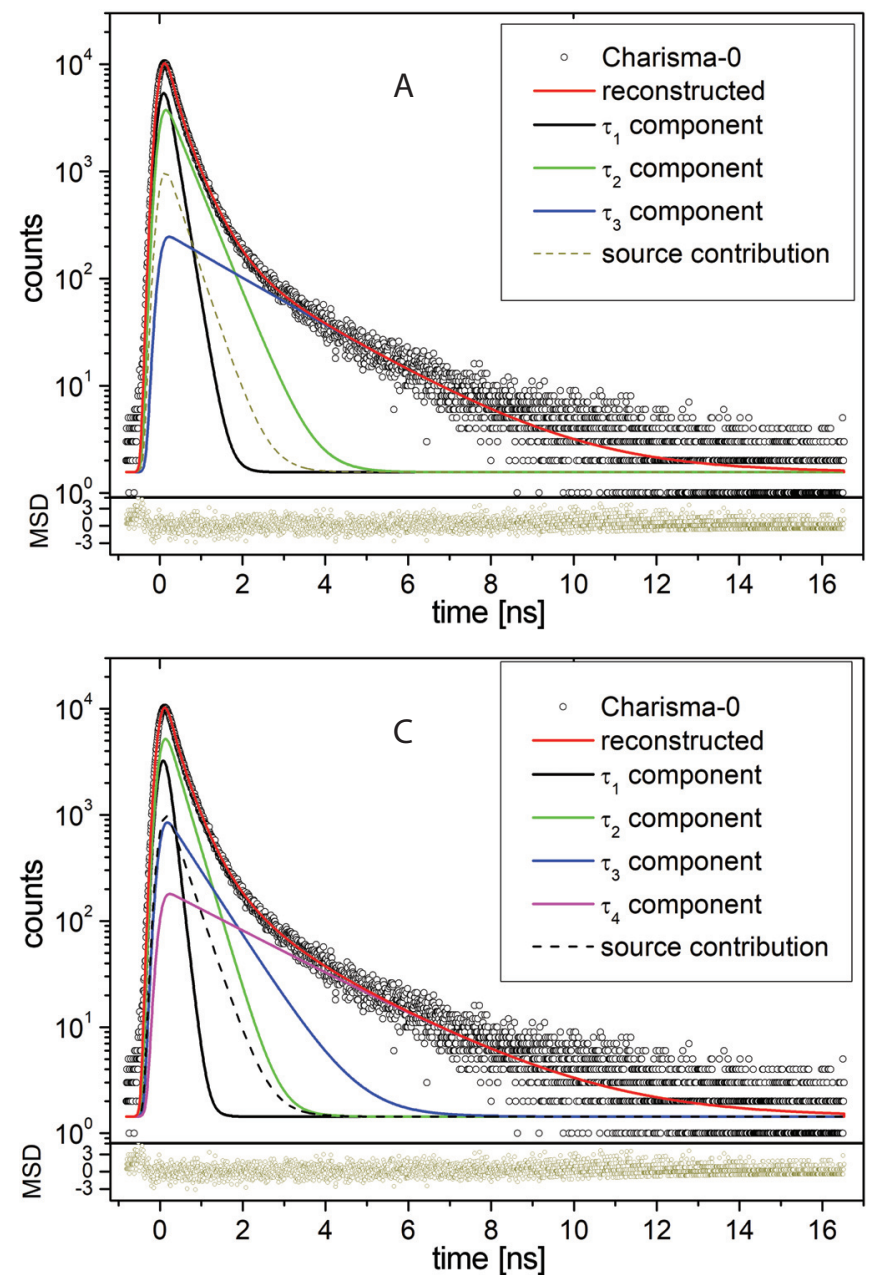

PAL spectra reconstructed from partially-constrained $\mathrm{x} 4$-term analysis are gathered in Tables 4 and 5 .

Numerous experiments during the recent decades testify that $\mathrm{x} 3$-term analysis is most commonly applied to reconstruct adequately raw PAL spectra for many inhomogeneous polymer/filler composites. ${ }^{14-21}$ Under such analysis (arranged as constraint-free decomposition ${ }^{14-20}$ or partially-constrained decomposition fixing the shortest $\tau_{1}$ lifetime $\left.{ }^{14,15,21}\right)$, the $3^{\text {rd }}$ component with a long-lived lifetime $\tau_{3}$ is ascribed to o-Ps annihilation in free-volume holes (voids), the $2^{\text {nd }}$ component with an intermediate lifetime $\tau_{2}$ is due to free positron annihilation in interfacial free volumes or other defect states mainly in a solid phase, and the $1^{\text {st }}$ component with the shortest lifetime $\tau_{1}$ is attributed to p-Ps self-annihilation conjugated with reduced $\mathrm{e}^{+}$annihilation from defect-free bulk state. ${ }^{4-6}$ The lifetime-fixing fitting is preferred to overcome inadequacy in the resolving of the shortest component due to mixing different annihilation events (especially, when $I_{1}$ intensity occurs to be substantially greater than $\left.I_{3}\right) \cdot{ }^{14,15}$ In such a case, the fixing $\tau_{1}$ to the value of $\mathrm{p}$-Ps lifetime improves the reliability of the finite-term analysis, not affecting the o-Ps lifetime. ${ }^{14}$ Nevertheless, unconstrained x3-decomposition has some, albeit limited, physical rel-
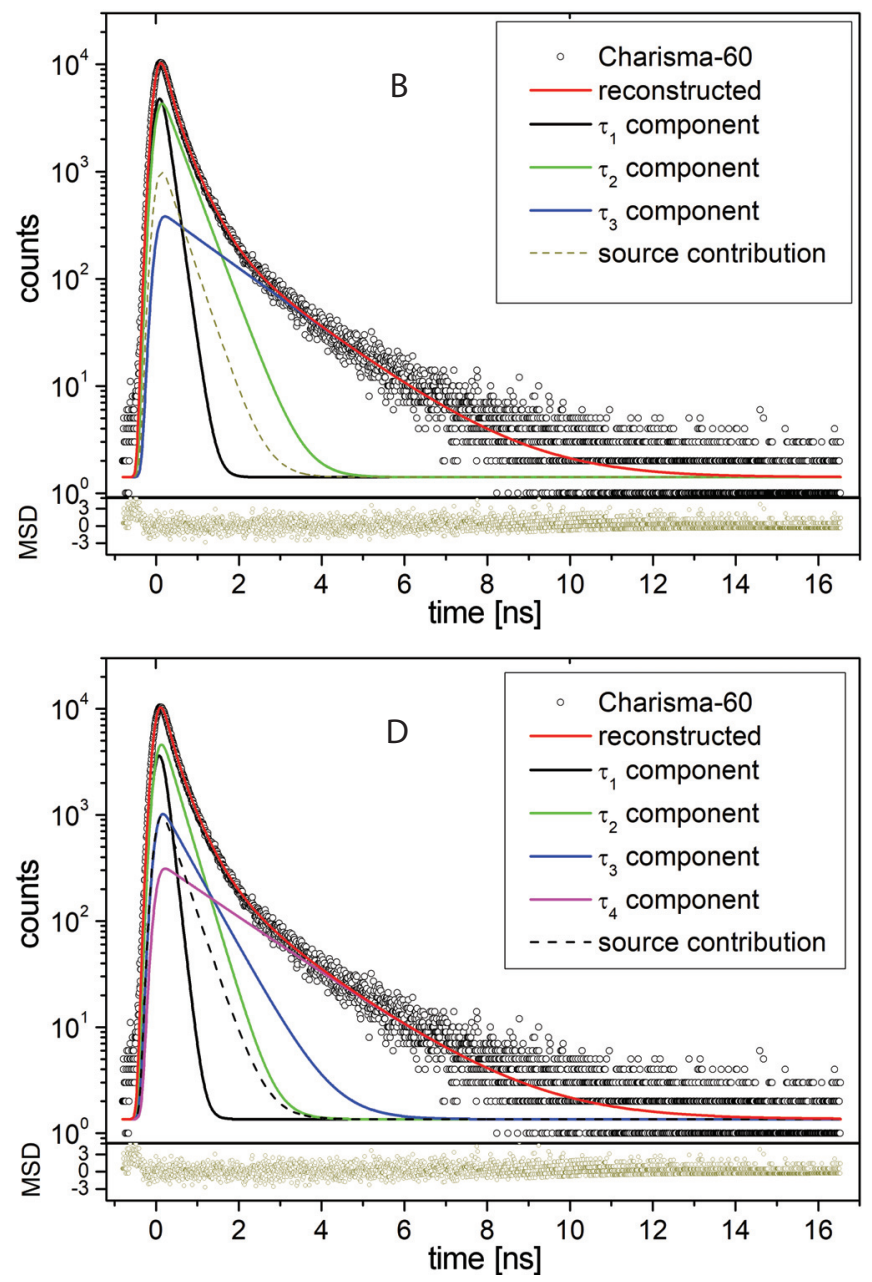

Fig. 1. Raw PAL spectra of non-polymerized Charisma-O (A, B) and photopolymerized Charisma-60 (C, D) DRC reconstructed from unconstrained x3-fitting (A, C) and partially-constrained $x 4$-fitting under fixed $\tau_{1}=0.125 \mathrm{~ns}(\mathrm{~B}, \mathrm{D})$ at the background of source contribution (the bottom insets show statistical scatter of variance) 

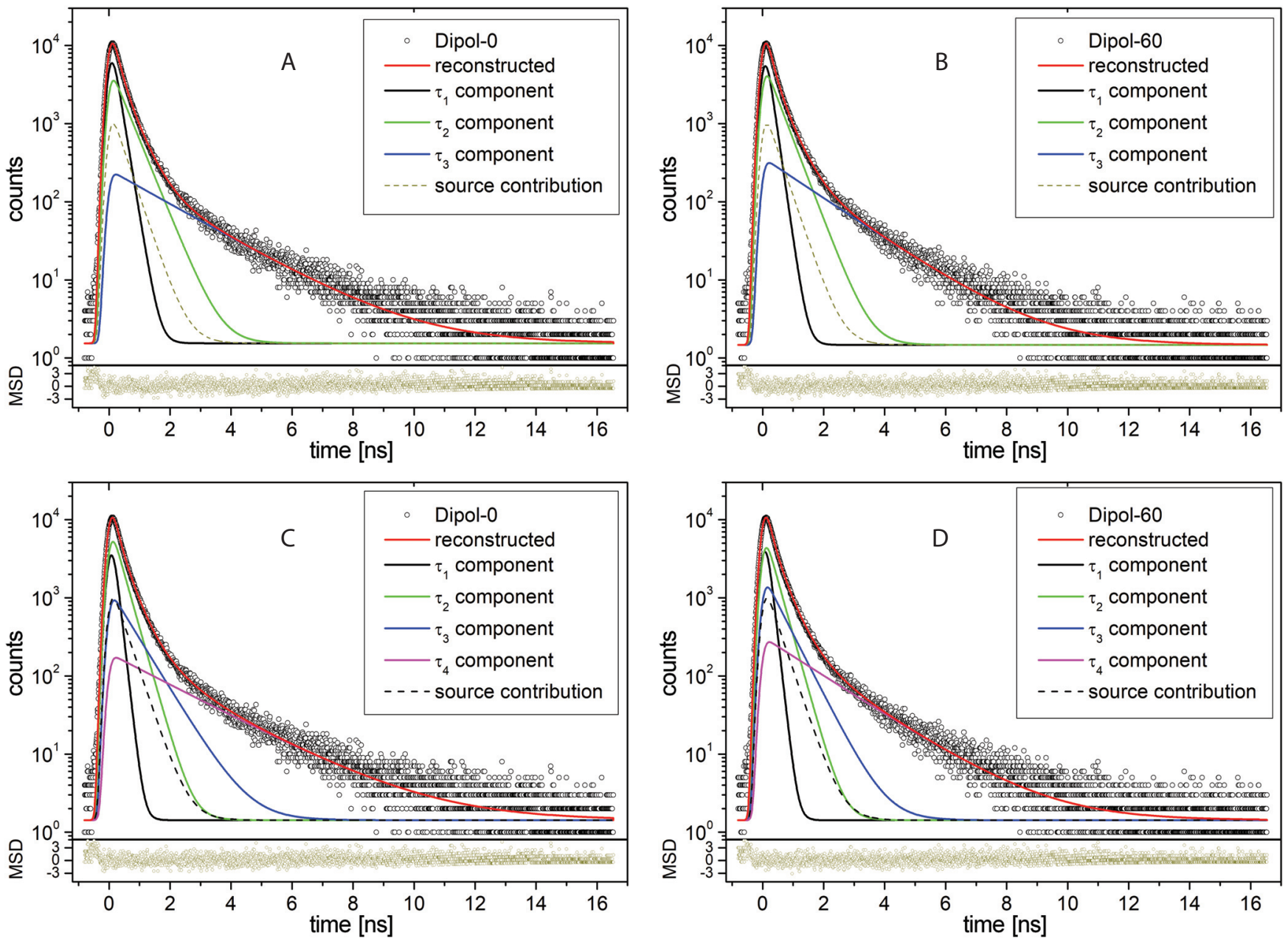

Fig. 2. Raw PAL spectra of non-polymerized Dipol-0 (A, B) and photopolymerized Dipol-60 (C, D) DRC reconstructed from unconstrained x3-fitting (A, C) and partially-constrained $x 4$-fitting under fixed $\tau_{1}=0.125 \mathrm{~ns}(B, D)$ in the background of source contribution (the bottom insets show statistical scatter of variance)

Table 1. PAL spectra fitting parameters for DRC within unconstrained x3-term decomposition

\begin{tabular}{|c|c|c|c|c|c|c|c|}
\hline \multirow{2}{*}{ DRC } & \multirow{2}{*}{ [FIT-1] } & \multicolumn{5}{|c|}{ PAL spectra fitting parameters } & \multirow{2}{*}{$\begin{array}{c}\tau_{\mathrm{av}} \\
{[\mathrm{ns}]}\end{array}$} \\
\hline & & $\tau_{1}[\mathrm{~ns}]$ & $\tau_{2}$ [ns] & $\tau_{3}[\mathrm{~ns}]$ & $\mathrm{I}_{2}$ [a.u.] & $\mathrm{I}_{3}$ [a.u. $]$ & \\
\hline Dipol-0 & 0.038 & 0.178 & 0.444 & 1.978 & 0.510 & 0.085 & 0.466 \\
\hline Dipol-60 & 0.034 & 0.155 & 0.403 & 1.601 & 0.560 & 0.103 & 0.442 \\
\hline Charisma-0 & 0.020 & 0.179 & 0.447 & 1.946 & 0.540 & 0.092 & 0.486 \\
\hline Charisma-60 & 0.008 & 0.158 & 0.415 & 1.560 & 0.560 & 0.110 & 0.458 \\
\hline
\end{tabular}

Table 2. PAL trapping models for DRC within unconstrained x3-term decomposition ignoring contributions from o-Ps decaying

\begin{tabular}{|c|c|c|c|c|c|c|c|c|}
\hline \multirow{2}{*}{ DRC } & \multicolumn{6}{|c|}{$\mathrm{e}^{+}$-trapping modes } & \multicolumn{2}{|c|}{ Ps-decaying modes } \\
\hline & $\tau_{\mathrm{av}}{ }^{\mathrm{tr}}[\mathrm{ns}]$ & $\tau_{b}[n s]$ & $\mathrm{K}_{\mathrm{d}}\left[\mathrm{ns}^{-1}\right]$ & $\tau_{2}-\tau_{b}[n s]$ & $\mathrm{\tau}_{2} / \tau_{\mathrm{b}}[\mathrm{a} . \mathrm{u}]$. & $\eta$ [a.u.] & $\mathrm{R}_{3}[\mathrm{~nm}]$ & $f_{v}^{3}[\%]$ \\
\hline Dipol-0 & 0.325 & 0.266 & 1.86 & 0.178 & 1.67 & 0.33 & 0.286 & 1.50 \\
\hline Dipol-60 & 0.309 & 0.251 & 2.45 & 0.152 & 1.61 & 0.38 & 0.248 & 1.17 \\
\hline Charisma-0 & 0.337 & 0.277 & 1.99 & 0.170 & 1.66 & 0.36 & 0.283 & 1.58 \\
\hline Charisma-60 & 0.321 & 0.260 & 2.50 & 0.155 & 1.60 & 0.40 & 0.243 & 1.19 \\
\hline
\end{tabular}

evance allowing the most stable fitting. ${ }^{14}$ Our previous results with some acrylic-type DRC also show that even under incomplete decomposition of the PAL spectra because of measuring instabilities, in part, in the vicinity of a $2^{\text {nd }}$ component originated from free $\mathrm{e}^{+}$annihilation, the best goodness of reconstruction is achieved under constraint-free $\mathrm{x} 3$-fitting. ${ }^{20}$ We also reconstructed the
Table 3. PAL trapping models for DRC within unconstrained x3-term decomposition assuming 2 additive positron-trapping defect states

\begin{tabular}{|l|c|c|c|c|}
\hline \multicolumn{1}{|c|}{ DRC } & $\tau_{\mathrm{av}}{ }^{\mathrm{tr}}[\mathrm{ns}]$ & $\tau_{\mathrm{b}}[\mathrm{ns}]$ & $\mathrm{K}_{\mathrm{d} 1}\left[\mathrm{~ns}^{-1}\right]$ & $\mathrm{K}_{\mathrm{d} 2}\left[\mathrm{~ns}^{-1}\right]$ \\
\hline Dipol-0 & 0.466 & 0.288 & 1.72 & 0.43 \\
Dipol-60 & 0.442 & 0.276 & 2.22 & 0.60 \\
Charisma-0 & 0.486 & 0.302 & 1.81 & 0.47 \\
Charisma-60 & 0.458 & 0.285 & 2.19 & 0.63 \\
\hline
\end{tabular}


Table 4. PAL spectra fitting parameters for DRC within partially-constrained $x 4$-term decomposition $\left(\tau_{1}=0.125 \mathrm{~ns}\right)$

\begin{tabular}{|c|c|c|c|c|c|c|c|c|}
\hline \multirow{2}{*}{ DRC } & \multirow{2}{*}[\mathrm{FIT}-1]{} & \multicolumn{6}{|c|}{ PAL spectra fitting parameters } & \multirow{2}{*}{$\begin{array}{c}\tau_{\mathrm{av}} \\
{[\mathrm{ns}]}\end{array}$} \\
\hline & & $\tau_{2}[n s]$ & $\tau_{3}[n s]$ & $\tau_{4}[\mathrm{~ns}]$ & $\mathrm{I}_{2}$ [a.u.] & $\mathrm{I}_{3}[$ a.u. $]$ & $\mathrm{I}_{4}$ [a.u.] & \\
\hline Dipol-0 & 0.059 & 0.331 & 0.699 & 2.199 & 0.563 & 0.146 & 0.068 & 0.465 \\
\hline Dipol-60 & 0.093 & 0.310 & 0.560 & 1.746 & 0.483 & 0.208 & 0.085 & 0.442 \\
\hline Charisma-0 & 0.021 & 0.346 & 0.799 & 2.275 & 0.601 & 0.132 & 0.066 & 0.487 \\
\hline Charisma-60 & 0.069 & 0.327 & 0.615 & 1.670 & 0.530 & 0.169 & 0.092 & 0.457 \\
\hline
\end{tabular}

Table 5. PAL trapping models for DRC within partially-constrained $x 4$-term decomposition $\left(\tau_{1}=0.125 \mathrm{~ns}\right)$

\begin{tabular}{|c|c|c|c|c|c|c|c|c|c|c|}
\hline \multirow{2}{*}{ DRC } & \multicolumn{6}{|c|}{$\mathrm{e}^{+}$-trapping modes } & \multicolumn{4}{|c|}{ Ps-trapping modes } \\
\hline & $\mathrm{T}_{\mathrm{av}}{ }^{\mathrm{tr}}[\mathrm{ns}]$ & $\tau_{\mathrm{b}}[\mathrm{ns}]$ & $\mathrm{K}_{\mathrm{d}}\left[\mathrm{ns}^{-1}\right]$ & $\tau_{2}-\tau_{b}[n s]$ & $\tau_{2} / \tau_{b}$ [a.u.] & $\eta$ [a.u.] & $\mathrm{R}_{3}[\mathrm{~nm}]$ & $\mathrm{f}_{\mathrm{v}}{ }^{3}[\%]$ & $\mathrm{R}_{4}[\mathrm{~nm}]$ & $\mathrm{f}_{\mathrm{v}}^{4}[\%]$ \\
\hline Dipol-0 & 0.272 & 0.225 & 3.55 & 0.106 & 1.47 & 0.44 & 0.107 & 0.14 & 0.306 & 1.47 \\
\hline Dipol-60 & 0.251 & 0.211 & 3.26 & 0.099 & 1.47 & 0.41 & 0.063 & 0.04 & 0.263 & 1.17 \\
\hline Charisma-0 & 0.290 & 0.239 & 3.82 & 0.107 & 1.45 & 0.48 & 0.130 & 0.22 & 0.313 & 1.51 \\
\hline Charisma-60 & 0.270 & 0.224 & 3.55 & 0.103 & 1.46 & 0.44 & 0.082 & 0.07 & 0.255 & 1.15 \\
\hline
\end{tabular}

PAL spectra with $x 3$-decomposition procedure fixing the background input. The results of such PAL spectra treatment along with fit goodness (not shown in Table 1) occurred to be very similar to those obtained under constraint-free $\mathrm{x} 3$-fitting.

With respect to the PAL data parameterized within constraint-free $\mathrm{x} 3$-term analysis (Tables 1-3), the annihilation process in both DRC can be identified as mixed $\mathrm{e}^{+}$-Ps-trapping, where "pure" o-Ps decaying is caused entirely by input from free-volume holes in the polymer matrix ( $3^{\text {rd }}$ component), while the $2^{\text {nd }}$ component is defined mainly by cumulative input from free $\mathrm{e}^{+}$-trapping sites in a filler (including interfacial free-volume holes between filler particles/nanoparticles). Typical radii of o-Ps-trapping sites in the polymer matrix of the studied DRC estimated from equation (7) slightly differ, approaching $3 \AA$ (2.86 $\AA$ in Dipol-0 and $2.83 \AA$ in Charisma-0), the corresponding fractional free volumes $f_{v}$ being $1.50 \%$ for $\mathrm{Di}$ pol-0 and 1.58\% $\AA$ for Charisma-0 DRC (Table 2). The same applies to $\mathrm{e}^{+}$-trapping channel defined by defect-related lifetimes close to $\tau_{2} \cong 0.45 \mathrm{~ns}$. However, strict parameterization of this channel in terms of a 2-state trapping model seems rather meaningless under essential input from o-Ps decays. ${ }^{4,6-8}$ The more realistic values of defectfree bulk lifetime $\tau_{b}$ related to positron annihilation from the Bloch states can be extracted from a simple model assuming additive $\mathrm{e}^{+}$-trapping inputs from trapped $\mathrm{e}^{+}$ and decayed o-Ps states (with lifetimes $\tau_{2}$ and $\tau_{3}$ given in Table 1). ${ }^{22}$ The calculated $\tau_{b}$ values (Table 3 ) occur to be nearly $10 \%$ overestimated compared to those presented in Table 2.

The Charisma ${ }^{\circledR}$ DRC is known to be composed of the finest filler particles of $\sim 10 \mathrm{~nm}$ pyrogenic silica $\mathrm{SiO}_{2}$ glass, which provide weight-packing density reaching $78 \%{ }^{11}$ The Dipol ${ }^{\circledR}$ DRC is structurally more variable, being composed of the finest filler particles of 1-3 nm, but smaller weight-packing density (72\%). ${ }^{12}$ As a result, the overall mass center of the PAL spectrum in non-polymerized Dipol-0 is shifted $\sim 20$ ps left as compared to Charisma-0
(Fig. 3). In spite of possible differentiation in filler size distribution, especially with respect to coarse-grained fraction, and variability in the finest filler fraction, these factors do not notably influence the PAL spectra shown in Fig. 1 and 2. This provides evidence for a similar intrinsic void structure responsible for $\mathrm{e}^{+}$- and Ps-trapping in these DRC. The numerical parameters of $\mathrm{e}^{-}$-Ps-trapping models in Charisma-0 and Dipol-0 DRC gathered in Table 2 and 3 can be accepted as a signature of their similar inner void structure, which concerns both the geometrical characteristics of free-volume voids (sizes of trapping sites) and their preferential chemical environment (composed of fine-grained $\mathrm{SiO}_{2}$ glass in a BisGMA-TEGDMAbased matrix).

In contrast, the $x 4$-term analysis of the PAL spectra could be ignored for both DRC, at least, in view of the worse best-fit goodness (compare the goodness of fitting (FIT-1) values in Tables 1 and 4). Nevertheless, this reconstruction procedure is often applied for some polymer/filler composites, especially when the basic polymer shows bifurcation in o-Ps lifetimes, as it occurs, for instance, in semi-crystalline polymers like polyethylene or polytetrafluoroethylene. ${ }^{14,15}$ In this case, the $4^{\text {th }}$ longestlived component is ascribed to o-Ps pick-off annihilation in holes of amorphous structure $\left(\tau_{4} \sim 2-4 \mathrm{~ns}\right)$, and the $3^{\text {rd }}$ intermediate component is ascribed to o-Ps pick-off annihilation in interstitial free-volume voids of the crystalline phase ( $\left.\tau_{3} \sim 1 \mathrm{~ns}\right)$. Therefore, in polytetrafluoroethylenebased composites filled with silica nanoparticles, the PAL spectra were well resolved in 4 discrete negative exponentials, but only the $4^{\text {th }}$ longest component was ascribed to o-Ps annihilation in a polymer matrix. ${ }^{23}$

Four discrete components were also resolved in the PAL spectra of some light-cured DRC based on dimethacrylate resins in other studies. ${ }^{24-26}$ Within a x4-fitting route assuming fixed $\tau_{1}$ value, the $4^{\text {th }}$ component was ascribed to o-Ps annihilation in the main part of the polymer matrix, while the $3^{\text {rd }}$ was explained as arising from o-Ps annihilation in the filler-matrix interphase. With re- 


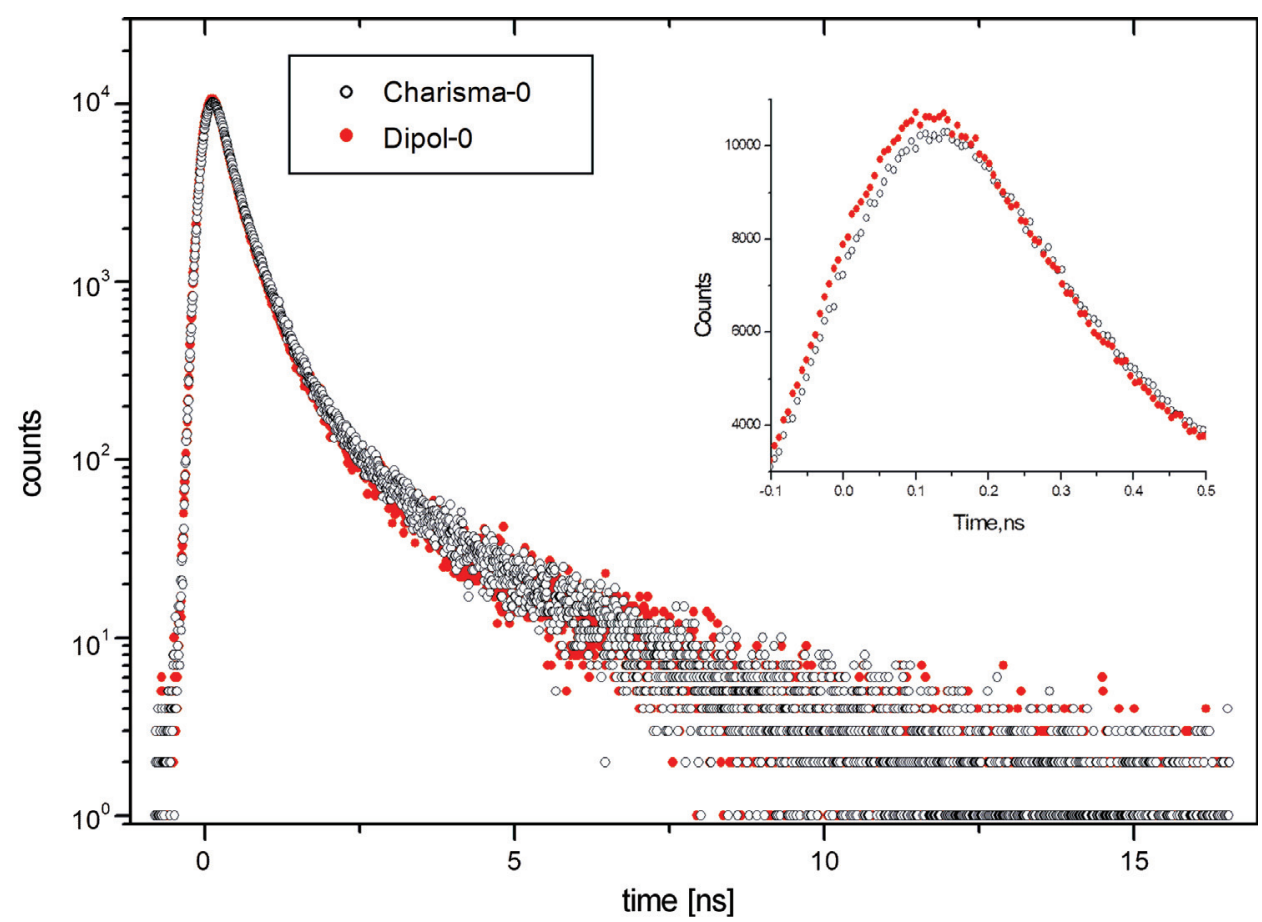

Fig. 3. Raw PAL spectra of non-polymerized Charisma-0 DRC as compared to that of Dipol-0 DRC (the inset shows a comparison of annihilation events accumulated in a peak)

spect to the latter, such an interpretation contradicts previous research, showing that only the $2^{\text {nd }}$ component with 0.3-0.5 ns lifetime (free $\mathrm{e}^{+}$annihilation) is responsible for trapping in interfacial free volumes. ${ }^{16-19}$ Recently, some current authors have shown that $x 4$-fitting assuming fixed shortest lifetime ( $\left.\tau_{1}=0.125 \mathrm{~ns}\right)$ was also favorable for dimethacrylate DRC subjected to long-term aging, which were initially characterized by x3-decomposed best-fitted PAL spectra. ${ }^{20,27}$ The origin of the additional component in o-Ps decaying in these polymer/filler DRC was not clarified unambiguously, while a version on stress-inducing destruction through growing inner and surface cracks seems quite plausible. It should be stressed that $\mathrm{x} 4$-term fitting parameters for Dipol ${ }^{\circledR}$ DRC affected by prolonged dry aging in the study mentioned above and non-polymerized Charisma-0 DRC in this research (Table 4) are very similar to the parameters for homemade DRC "Composite 16 " in other studies..$^{20,24,25}$ Of note, all these DRC possess a polymer matrix of high crosslink density composed of a mixture of BisGMA and TEGDMA monomers.

In cases above, simple physical mixing in the $2^{\text {nd }}$ component of $x 4$-term decomposed PAL spectra due to interfacial holes and other alternatives (free-volume defects in the solid/polymer phase) cannot be excluded in separation separating the most realistic annihilation channels. This analysis may provide invalid parameterization of the $2^{\text {nd }}$ component as an artifact of the inadequate $x 4$-fitting route, since the polymer matrices of DRC based on bisphenol A polycarbonates are solely characterized by x3-term PAL spectra, where only a long-lived component comes undoubtedly from o-Ps decaying. ${ }^{28-30}$ In the case of multiple o-Ps decays of the same origin, this component can be easily replaced by apparent lifetime, which is a mean value averaged over all o-Ps components with corresponding intensities. $^{30}$

The most essential difference concerns the photopolymerization effect on the PAL trapping modes, revealed in the modulation depth for initial (non-cured) and final (light-cured) DRC.

Due to structural variability in the finest filler fraction, Dipol $^{\circledR}$ DRC is known to possess relatively small photopolymerization volumetric shrinkage of $2.2 \%$, while in Charisma ${ }^{\circledR}$ DRC this macroscopic parameter approaches $2.9 \%{ }^{11,12}$ Thus, the volumetric shrinkages in these DRC are respectively rationed as $\sim 1.3$. Despite the eventual difference in the selectivity of the PAL method to different $\mathrm{e}^{+}$-Ps trapping sites (not only those which determine macroscopic shrinkage), we suggest estimating this ratio based on free-volume void parameters extracted from x3-term PAL data (Table 2 and 3).

Thus, with respect to o-Ps trap sizes determined from $\tau_{3}$ lifetime using equation (7), the spherical void volume in Dipol ${ }^{\circledR}$ DRC drops by $34.8 \%$ (from $93.6 \AA^{3}$ to $61.0 \AA^{3}$ ). In Charisma ${ }^{\circledR} \mathrm{DRC}$, this decrease is deeper, reaching nearly $40 \%$ (from $90.7 \AA^{3}$ to $54.4 \AA^{3}$ ). Thus, the corresponding ratio is close to 1.15. A similar value (1.12) can be obtained from photopolymerized dropping in fractional free volumes $f_{v}$. Therefore, the o-Ps traps themselves do not cover the overall macroscopic shrinkage in the studied DRC.

The similar calculations for a "pure" $\mathrm{e}^{+}$-trapping channel defined from the $2^{\text {nd }}$ component in the $\mathrm{x} 3$-term PAL spectra has no strong physical meaning, provided essential input from the $3^{\text {rd }}$ component (related to o-Ps decaying). ${ }^{4,5}$ Nevertheless, we shall perform such an estimation 
ignoring this specificity, which can be admitted under a supposition on similar disturbance in the $\mathrm{e}^{+}$-trapping channel from o-Ps traps in both DRC samples. Thus, the decrease in defect-related $\tau_{2}$ lifetime (which reflects the size of respective $\mathrm{e}^{+}$traps) reaches $9.2 \%$ in $\mathrm{Dipol}^{\circledR}$ (from 0.444 ns to 0.403 ns; Table 2) and $7.2 \%$ in Charisma ${ }^{\circledR}$ (from $0.447 \mathrm{~ns}$ to $0.415 \mathrm{~ns}$ ), giving an opposite effect in comparison, reaching $\sim 0.8 .^{4-6}$ Such inconsistency is quite understandable in view of inadequacy in the interpretation of the $2^{\text {nd }}$ component intensity $I_{2}$ in the $x 3$-term decomposed PAL spectrum (which does not reflect solely the content of "pure" $\mathrm{e}^{+}$traps, but also balancing in the mixed $\mathrm{e}^{+}$-Ps-trapping channels as a complement to full intensity normalization). Indeed, in contrast to a polymerization-induced decrease in fractional free-volume $f_{v}$ for o-Ps, the fraction $\eta$ of trapped $\mathrm{e}^{+}$reveals an enormous compensating increase of $15.2 \%$ in Dipol $^{\circledR}(0.33-0.38$; Table 2) and $11.1 \%$ in Charisma ${ }^{\circledR}$ DRC $(0.36-0.40)$, thus giving it a ratio $\sim 0.7$.

Under a condition of mixed $\mathrm{e}^{+}$-Ps-trapping channels, especially when these channels are inter-connected, the most adequate estimation of volumetric shrinkage independently of the computer-fitting procedure can be done using average $\tau_{a v}$ lifetime, e.g., mass center of PAL spectrum defined with respect to equation (1). As it is shown in Table 1 , the light-curing results in $\tau_{a v}$ decrease, this effect reaching $5.1 \%$ for Dipol ${ }^{\circledR}$ (from 0.466 to 0.442 ns) and $5.8 \%$ for Charisma ${ }^{\circledR}$ DRC (from 0.486 to $0.458 \mathrm{~ns}$ ). These values give $\sim 1.2$ in ratio, which can be accepted as being in very good accord with a rougher macroscopic estimation $(\sim 1.3)$.

Due to the trapping parameters defined from unconstrained x3-term decomposition (Table 1-3), the photopolymerization volumetric shrinkage in the DRC is well revealed through a decrease in average positron lifetime $\tau_{a v}$, this effect being accompanied by changes in both o-Ps- and $\mathrm{e}^{+}$-trapping channels. Indeed, the photopolymerized DRC possess reduced long-lived lifetimes $\tau_{3}$, but increased $I_{3}$ intensities (Table 1), thus resulting in smaller fractional free volumes $f_{v}$ (Table 2). These changes are dominated in a light-cured state, being partially overbalanced by increased fraction $\eta$ of trapped $\mathrm{e}^{+}$(Table 2 ), resulting from higher $I_{2}$ intensities and slightly suppressed $\tau_{2}$ lifetimes (Table 1 ). The observed changes in o-Ps-trapping models can be ascribed preferentially to cross-linking of structural chains dominated in a "pure" polymer matrix, like it occurs in other polymers under UV light exposure, vulcanization (due to thermal curing) or $\gamma$-irradiation. ${ }^{31-33}$ In fact, this causes a greater number of smaller voids in the photopolymerized DRC, thereby resulting in free-volume fragmentation for both existing o-Ps- and $\mathrm{e}^{+}$-trapping sites, as illustrated by the schematic cartoon in Fig. 4. This global fragmentation trend can be well revealed in the increased trapping rates $\kappa_{d 1}$ and $\kappa_{d 2}$ for both DRC Dipol-60 and Charisma-60, defined within unconstrained $\mathrm{x} 3$-term fitting assuming 2 additive positron-trapping states arise from distinguished $\mathrm{e}^{+}$- and Ps-trapping channels (Table 3).
By assuming that modification changes in o-Ps and $\mathrm{e}^{+}-$ trapping sites under light curing are mutually interconnected, so that no effects occur in other annihilation channels (such as annihilation from defect-free bulk states, or generation of novel Ps- or $\mathrm{e}^{+}$traps), we can treat the data within a x3-x2-coupling decomposition algorithm. ${ }^{34,35}$ This approach concerns unconstrained $x 3$-term reconstructed PAL spectra transformed to generalized x2-term form for host (non-polymerized) and modified (lightcured or photopolymerized) DRC, where a $2^{\text {nd }}$ component involves contributions from all possible trapping inputs (e.g., $\mathrm{e}^{+}$traps, input from o-Ps decaying and p-Ps self-annihilation). Such simplification makes it possible to resolve additional components in the generalized x2-term PAL spectrum for modified matrix (with lifetime $\tau_{\text {int }}$ and intensity $\left.I_{\text {int }}\right)$, the compensating $\left(\tau_{n}, I_{n}\right)$ input in the $1^{\text {st }}$ channel being found assuming a reasonable condition of full interchannel equilibrium. ${ }^{33-37}$ Thereby, the parameterization of transformed Ps-e $\mathrm{e}^{+}$traps in the photopolymerized DRC matrix can be performed accepting $\left(\tau_{n}, I_{n}\right)$ and $\left(\tau_{\text {int }}, I_{\text {int }}\right)$ as the respective $1^{\text {st }}$ and $2^{\text {nd }}$ components of the generalized x2-term PAL spectrum for some hypothetical medium obeying parameterization, with respect to formalization of a simple 2-state positron-trapping model. ${ }^{4-8}$ The defectrelated $\tau_{\text {int }}$ positron lifetime in this model reflects appearing/disappearing traps in dependence on a positive/negative sign of $I_{n}$ and $I_{\text {int }}$ intensities.

The calculated trapping parameters of the studied lightcured DRC determined with respect to non-polymerized ones using the $\mathrm{x} 3$-x2-coupling decomposition algorithm are given in Table $6.34,35$ The negative $I_{n}$ and $I_{\text {int }}$ intensities testify that the parameterization concerns disappearing traps, which are rather like o-Ps free-volume holes with respect to $\tau_{\text {int }}$ values $(0.656 \mathrm{~ns}$ for Charisma-60 and $0.768 \mathrm{~ns}$ for Dipol-60 DRC), exceeding the character level of o-Ps and p-Ps self-annihilation in a vacuum $(0.5 \mathrm{~ns}) .4$
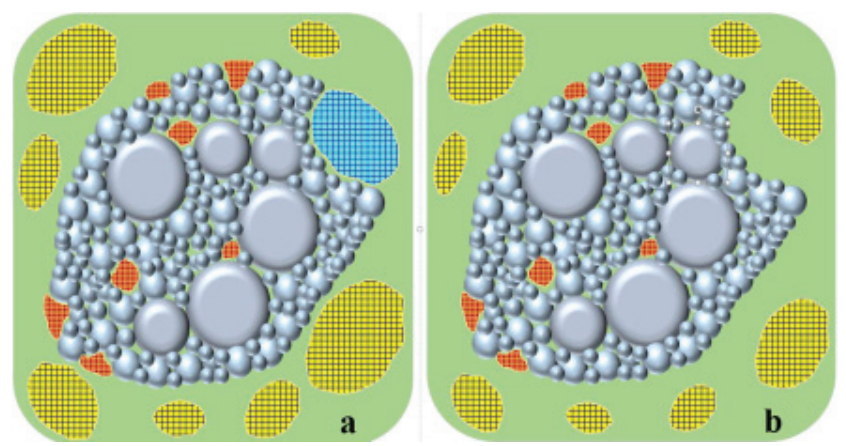

Fig. 4. Schematic cartoon showing fragmentation of free-volume Ps and $\mathrm{e}^{+}$-traps in light-cured DRC: a - microstructure fragment of agglomerated filler particles (grey-colored) in non-polymerized DRC matrix (greencolored) containing o-Ps-trapping void located in interfacial filler-polymer region (blue-cross-dashed), o-Ps-trapping holes preferentially in polymer matrix (yellow-cross-dashed) and $\mathrm{e}^{+}$-trapping sites preferentially in filler matrix (red-cross-dashed); $b$ - the same agglomerate of filler particles in fully polymerized DRC matrix (o-Ps-trapping void in the interfacial fillerpolymer region disappears, giving rise to more contracted o-Ps-trapping holes in surrounding polymer matrix and reduced trapping sites within agglomerated filler particles). 
Table 6. PAL trapping models for light-cured DRC determined within $\times 3$-x2-coupling decomposition algorithm ${ }^{34,35}$

\begin{tabular}{|c|c|c|c|c|c|c|c|}
\hline \multirow{2}{*}{ DRC } & \multicolumn{2}{|c|}{$1^{\text {st }}$ component } & \multicolumn{2}{|c|}{$2^{\text {nd }}$ component } & \multicolumn{3}{|c|}{ Trapping modes } \\
\hline & $\tau_{\mathrm{n}}[\mathrm{ns}]$ & $\ln [$ a.u. $]$ & $\tau_{\text {int }}[\mathrm{ns}]$ & $\mathrm{l}_{\text {int }}[$ a.u. $]$ & $\mathrm{T}_{\mathrm{av}}[\mathrm{ns}]$ & $\tau_{b}[n s]$ & $\mathrm{K}_{\mathrm{d}}\left[\mathrm{ns}^{-1}\right]$ \\
\hline Charisma-60 & 0.409 & -0.027 & 0.656 & -0.086 & 0.597 & 0.573 & 0.70 \\
\hline Dipol-60 & 0.542 & -0.018 & 0.768 & -0.056 & 0.713 & 0.698 & 0.41 \\
\hline
\end{tabular}

These traps are located preferentially at the grain boundaries of agglomerated filler particles (i.e., in the interfacial filler-polymer region possessing lower space occupation, Fig. 4a), as it follows from the sufficiently high defect-free bulk positron lifetime $\tau_{b}$ in Table 6 (0.597 ns for Charisma-60 and 0.713 ns for Dipol-60 DRC). In the due course of photopolymerization, these interfacial Ps-trapping sites disappear, giving rise to more contracted Ps traps (free-volume holes) in the surrounding polymer matrix and reduced trapping sites within agglomerated filler particles (Fig. 4b).

\section{Conclusions}

The characterization possibilities of positron annihilation lifetime spectroscopy are analyzed as they apply to 2 commercially available dimethacrylate-type dental restorative composites, Charisma ${ }^{\circledR}$ and Dipol ${ }^{\circledR}$, both based on a monomer matrix consisting of bisphenol A-diglycidyl dimethacrylate (BisGMA) and triethylene glycol dimethacrylate (TEGDMA) modified with multisized filler particles having a highly dispersive phase of silica glass. These composites were studied in the initial and deeply light-cured states, using a conventional fast-fast coincidence positron lifetime spectrometer equipped with ORTEC ${ }^{\circledR}$ electronics. The PAL spectra were reconstructed from unconstrained $\mathrm{x} 3$-term and partially-constrained $\mathrm{x} 4$-term fitting routes, assuming the shortest lifetime fixed at a theoretical value of intrinsic para-Ps self-annihilation $(0.125 \mathrm{~ns})$.

With respect to the data parameterized within free $\mathrm{x} 3$-term analysis, the annihilation in the composites is identified as mixed positron-Ps trapping, where o-Ps decaying is caused entirely by free-volume holes in the polymer matrix, and the $2^{\text {nd }}$ component is defined mainly by interfacial free-volume holes between the filler particles and surrounding polymer. Typical radii of o-Ps-trapping sites in a composite polymer matrix slightly differ, approaching $3 \AA$, and fractional free volumes reach $1.50 \%$ for Dipol ${ }^{\circledR}$ and $1.58 \% \AA$ for Charisma ${ }^{\circledR}$. The partially-constrained $\mathrm{x} 4$-term analysis of lifetime spectra is less efficient, giving greater scatter of variance with an artifact of fixed shortest lifetime, allowing unresolved physical mixing in the $2^{\text {nd }}$ component. The most adequate model-independent estimation of polymerization volumetric shrinkage in the studied composites under condition of mixed positron-Ps trapping can be done in terms of average positron lifetime. The meaningful description of transformations in Ps and positron-trapping sites under light curing, which occurs more efficiently in the Charisma ${ }^{\circledR}$ than in the Dipol $^{\circledR}$ composites, can be developed on the basis of a semiempirical model exploring x3-x2-coupling decomposition algorithm..$^{34,35}$

\section{References}

1. Cramer NB, Stansbury JW, Bowman CN. Recent advantages and developments in composite dental restorative materials. J Dent Res. 2011;90:402-416.

2. Ferracane JL. Resin composite - state of the art. Dent Mater. 2011;27:29-38.

3. Rühle M, Dosch H, Mittemeijer EJ, Van de Voorde MH. European White Book on Fundamental Research in Materials Science. Stuttgart: Max-Planck-Institut für Metallforschung; 2002.

4. Krause-Rehberg R, Leipner H. Positron Annihilation in Semiconductors: Defect Studies. Heidelberg: Springer; 1999.

5. Jean YC. Positron annihilation spectroscopy for chemical analysis: A novel probe for microstructural analysis of polymers. Microchem J. 1990;42:72-102.

6. Shpotyuk O, Filipecki J. Free Volume in Vitreous Chalcogenide Semiconductors: Possibilities of Positron Annihilation Lifetime Study. Czestochowa, Poland: Ed. WSP; 2003.

7. Keeble DJ, Brossmann U, Puff W, Würschum R. Positron annihilation studies of materials. In: Kaufmann EN, ed. Characterization of Materials. Hoboken, NJ: John Wiley \& Sons; 2012:1899-1925.

8. Tuomisto F, Makkonen I. Defect identification in semiconductors with positron annihilation: Experiment and theory. Rev Mod Phys. 2013;85:1583-1631.

9. Jean YC, Van Horn JD, Hung WS, Lee KR. Perspective of positron annihilation spectroscopy in polymers. Macromolecules. 2013;46:7133-7145.

10. Mitra SB, Wu D, Holmes BN. An application of nanotechnology in advanced dental materials. J Am Dent Assoc. 2003;34:1382-1390.

11. Charisma ${ }^{\circledR}$. Scientific Information. Heraeus Kulzer GmbH, Hanau, Germany, http://pantelides-dental.gr/userfiles/files/CharismaScientificlnformation.pdf. Accessed April 11, 2018.

12. Dipol ${ }^{\circledR}$. Composite Universal. Instruction on using Dipol materials. Oksomat-AN, Ukraine Dental Products, 6-7.

13. Kansy J. Microcomputer program for analysis of positron annihilation lifetime spectra. Nucl Instrum Methods Phys Res A. 1996;374:235-244.

14. Dlubek G, Clarke AP, Fretwell HM, Dugdale SB, Alam MA. Positron lifetime studies of free volume hole size distribution in glassy polycarbonate and polystyrene. Phys Stat Sol. 1996;A157:351-364.

15. Dlubek G, Saarinen K, Fretwell HM. Positron states in polyethylene and polytetrafluoroethylene: A positron lifetime and Dopplerbroadening study. Nucl Instrun Methods Phys Res B. 1998;142:139-155.

16. Wang SJ, Wang CL, Zhu XG, Qi ZN. Structural characteristics of HDPE/CaCO3 polymer composites probed by positron annihilation. Phys Stat Sol. 1994;A142:275-280.

17. Zhang M, Fang PF, Zhang SP, Wang B, Wang SJ. Study of structural characteristics of $\mathrm{HDPE} / \mathrm{CaCO}_{3}$ nanocomposites by positrons. Rad Phys Chem. 2003;68:565-567.

18. Jia S, Zhang Z, Fan Y, Weng H, Zhang X, Hang R. Study of the size and numerical concentration of the free volume of carbon filled HDPE composites by the positron annihilation method. Eur Polym J. 2002;38:2433-2439.

19. Awad S, Chen HM, Grady BP, et al. Positron annihilation spectroscopy of polystyrene filled with carbon nanomaterials. Macromolecules. 2012;45:933-940. 
20. Boyko O, Shpotyuk Y, Filipecki J. Positron annihilation lifetime study of extended defects in semiconductor glasses and polymers. Phys Stat Sol C. 2013;10:121-124.

21. Jobando VO, Quarles CA. Positron lifetime studies on the free volume changes during curing of rubber-carbon black composites. Phys Stat Sol C. 2007;4:3763-3766.

22. Liu M, Kitai AH, Mascher P. Point defects and luminescence centers in zinc oxide and zinc oxide doped with manganese. J Lumin. 1992:54:35-42.

23. Madami MM, MacQueen RC, Granata RD. Positron annihilation lifetime study of PTFE/silica composites. J Polym Sci B. 1996;34:2767-2770.

24. Kleczewska J, Bieliński DM, Dryzek E, Piątkowska A. Application of positron annihilation lifetime spectroscopy in studies of dental composites based on dimethacrylate resins. In: Pielichowski K, ed. Modern Polymeric Materials for Environmental Application. Vol. 4(1), Kraków, Poland: TEZA; 2010:143-150.

25. Kleczewska J, Bieliński DM, Ranganathan N, Sokołowski J. Characterization of light-cured dental composites. In: Ranganathan N, ed. Materials Characterization. Modern Methods and Applications. Boca Raton, FL: CRC Press Taylor \& Francis Group; 2016:117-148.

26. Shirazinia M, Mehmandoost-Khajeh-Dad AA, Dehghani V, Mehmandoost-Khajeh-Dad J, Khaghani M. The effect of curing light intensity on free volume size in some dental composites. Polim Med. 2016;46:129-133.

27. Filipecki J, Chamerski K, Boyko O, Kotynia K. Ageing phenomenon in acrylic polymer dental materials detected by means of positron annihilation lifetime spectroscopy. Polim Med. 2014;44:21-28.

28. Pfeifer CS, Shelton ZR, Braga RR, Windmoller D, Machalo JC, Stansbury JW. Characterization of dimethacrylate polymeric networks: A study of the crosslinked structure formed by monomers used in dental composites. Eur Polym J. 2011;47:162-170.

29. Kluin JE, Yu Z, Vleeshouwers S, McGervey JD, Jamieson AM, Simha R. Temperature and time dependence of free volume in bisphenol $A$ polycarbonates studied by positron lifetime spectroscopy. Macromolecules. 1992;25:5089-5093.

30. Kluin JE, Yu Z, Vleeshouwers S, et al. Ortho-positronium lifetime studies of free volume in polycarbonates of different structures: Influence of hole size distribution. Macromolecules. 1993;26:1853-1861.

31. Ramani R, Ranganathaiah C. Degradation of acrylonitrile-butadiene-styrene and polycarbonate by UV irradiation. Polym Degrad Stab. 2000;69:347-354.

32. Srithawatpong R, Peng ZL, Olson BG, et al. Positron annihilation lifetime studies of changes in free volume on cross-linking cispolyisoprene, high-vinyl polybutadiene, and their miscible blends. J Polym Sci B. 1999;37:2754-2570.

33. Hyla M, Filipecki J, Swiatek J, Mervinskii RI. Gamma irradiation effects on UV-cured polymers based on acrylate oligomers studied by positron annihilation lifetime spectroscopy. J Non-Cryst Solids. 2005;351:1473-1476.

34. Shpotyuk O, Filipecki J, Ingram A, et al. Positronics of subnanometer atomistic imperfections in solids as a high-informative structure characterization tool. Nanoscale Res Lett. 2015;10:77-1-5.

35. Shpotyuk O, Ingram A, Filipecki J, Bujňáková Z, Baláž P. Positron annihilation lifetime study of atomic imperfections in nanostructurized solids: On the parameterized trapping in wet-milled arsenic sulfides As4S4. Phys Stat Sol. 2016;B253:1054-1059.

36. Shpotyuk O, Ingram A, Shpotyuk O. Free volume structure of acrylic-type dental nanocomposites tested with annihilating positrons. Nanoscale Res Lett. 2016;11:528-1-6.

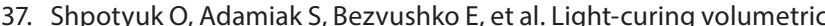
shrinkage in dimethacrylate-based dental composites by nanoindentation and PAL study. Nanoscale Res Lett. 2017;12:75-1-6. 